\title{
Degradación de materiales cerámicos en atmósferas de combustión. Dos casos prácticos: quemadores y turbinas de gas
}

\author{
E. GARCÍA, M.I. OSENDI, P. MIRANZO \\ Instituto de Cerámica y Vidrio (CSIC)
}

\begin{abstract}
El buen comportamiento de los materiales cerámicos en ambientes corrosivos y a altas temperaturas los sitúa, preferentemente, en todos aquellos procesos industriales que buscan el ahorro energético, en particular los procesos de combustión de gases o de derivados del petróleo. Sin embargo, las atmósferas de combustión que se producen en ciertas aplicaciones producen la degradación de estos materiales. En este trabajo se estudian dos ejemplos de materiales cerámicos empleados en este tipo de aplicaciones; un material de cordierita, empleado como quemador de gas radiante para su posible aplicación en calderas de combustión domésticas y un recubrimiento de $\mathrm{ZrO}_{2}$ estabilizado con $\mathrm{Y}_{2} \mathrm{O}_{3}$, empleado como barrera térmica en componentes de una turbina de gas para la producción de energía eléctrica. El estudio de la microestructura, la composición química y de fases cristalinas de ambos materiales después de su degradación en uso permite determinar cuales son los mecanismos que han tenido lugar y qué elementos de la atmósfera de trabajo han sido los causantes de dicha degradación.
\end{abstract}

Palabras clave: Cordierita, $\mathrm{Y}_{\mathrm{ZrO}} \mathrm{Zr}^{\prime}$ Quemadores, Recubrimientos, Barreras Térmicas, Corrosión.

Degradation of ceramic materials in combustion environments: two practical cases: gas burners and turbines

The use of ceramic materials in energy applications with the aim of power saving through the use of high working temperatures is widely extended due to their better behaviour in those working conditions compared to than metallic materials. However, the combustion environments developed in specific applications can lead to the degradation of such materials. In this work, two ceramic materials employed in these applications are studied; a cordierite material, used as gas radiant burner for its possible application in domestic combustion boilers, and an $\mathrm{Y}_{2} \mathrm{O}_{3}$ stabilized $\mathrm{ZrO}_{2}$ coating, used as thermal barrier coating on metallic components of a gas turbine for power generation. The microstructure and phase evolution in both materials after ageing under working conditions allows identifying the main degradation mechanism responsible of the degradation.

Keywords: Cordierite, $\mathrm{Y} \mathrm{ZrO}_{2^{\prime}}$ Burners, Thermal Barrier Coatings, Corrosion.

\section{INTRODUCCIÓN}

La preocupación por la conservación del medio ambiente se ha convertido en un tema de interés desde hace pocas décadas. Se ha demostrado que el uso de combustibles fósiles tiene una gran contribución al efecto invernadero, causado principalmente por la emisión de $\mathrm{CO}_{2}$. Además, en el quemado de combustibles, también se generan otros contaminantes, como los $\mathrm{NO}_{x}\left(\mathrm{NO}, \mathrm{NO}_{2}\right.$ y $\left.\mathrm{N}_{2} \mathrm{O}\right)$ y $\mathrm{SO}_{x}\left(\mathrm{SO}, \mathrm{SO}_{2}\right)$. No obstante, la demanda de energía térmica es cada vez mayor, lo que hará que los combustibles derivados del petróleo sigan teniendo importancia en las próximas décadas, aunque el desarrollo de energías renovables, como la solar o eólica, sea cada vez más importante. Como consecuencia de esta demanda, el quemado de combustibles líquidos como el aceite, el gasoil o la gasolina, tradicionalmente "sucios" por la presencia de contaminantes adicionales como sodio, potasio, vanadio o fósforo, está evolucionado hacia procesos más limpios mediante el uso de catalizadores $(1,2)$ y la mejora de la eficiencia de los procesos de combustión (3).

Por otra parte, la combustión de gas natural es muy común en aplicaciones domésticas e industriales, como los sistemas centrales de calefacción, las cocinas, las turbinas de gas y más recientemente las microturbinas de gas para la generación de electricidad. Una ventaja del gas natural es su mayor limpieza, en comparación con otros combustibles, ya que no hay emisión de $\mathrm{SO}_{\mathrm{x}}$ debido a que todos los compuestos de azufre son eliminados del gas natural antes del transporte. Sin embargo, sí se producen emisiones de $\mathrm{CO}_{2}, \mathrm{CO}$ y NO . $\mathrm{El} \mathrm{CO}_{2}$ es inherente al quemado de combustibles fósiles y solamente puede reducirse disminuyendo la cantidad de combustible quemado. La emisión de $\mathrm{CO}$ es pequeña cuando se consigue una combustión eficiente. La emisión de $\mathrm{NO}_{x}$ es inherente a la combustión con aire y se puede reducir si la temperatura de los gases de combustión decrece. Este objetivo se puede alcanzar usando quemadores de superficie que trabajen en modo radiante, ya que la llama se enfría en contacto con el quemador que emite esta energía principalmente por radiación. En este aspecto, los quemadores fabricados con materiales porosos han demostrado ser los más idóneos $(4,5)$. El material usado en la construcción del quemador es importante pues la cantidad total de calor irradiado es dependiente de la naturaleza de su superficie y de su temperatura. Los requisitos principales que debe cumplir un material para que sea adecuado como 
quemador son: bajo coeficiente de dilatación térmica y baja conductividad térmica, alta emisividad térmica en la superficie de combustión, estabilidad mecánica a alta temperatura, y una porosidad elevada para que aumente tanto la eficiencia de la combustión como la superficie radiante total. Además, existen requerimientos comerciales como que deban ser económicos, disponibles en poco tiempo y fáciles de producir industrialmente. Los materiales cerámicos son los más indicados para este tipo de aplicación (6).

De igual forma, los materiales cerámicos han estado presentes en el desarrollo de la tecnología de los motores de turbina (7). Un claro ejemplo es el $\mathrm{ZrO}_{2}$ estabilizado con $7 \%$ en peso de $\mathrm{Y}_{2} \mathrm{O}_{3}$ (YSZ), que ha sido utilizado de forma extensiva como barrera térmica (Thermal Barrier Coatings o TBCs, en la literatura inglesa) para proteger los componentes metálicos de las altas temperaturas de los gases calientes que se generan en los motores de turbina, utilizados en aviación y en la generación de electricidad. El uso de TBCs de espesores entre 100 y $500 \mu \mathrm{m}$ reduce la temperatura superficial de los componentes metálicos hasta $300{ }^{\circ} \mathrm{C}$, pudiendo de esta forma incrementar la temperatura de la cámara de combustión (8-10). Esto aumenta la eficiencia del proceso de combustión y reduce la cantidad de combustible necesaria para producir la misma cantidad de energía y, por tanto, reduce la emisión de gases contaminantes.

Los materiales cerámicos usados en este tipo de aplicaciones están sometidos a distintos procesos de degradación producidos por las altas temperaturas y los ciclos térmicos a los que se ven sometidos, así como por la acción de especies químicas corrosivas, subproductos de la combustión.

La corrosión es la alteración física y química de un material como resultado de su interacción con el ambiente en el que trabaja. Uno de los parámetros que se usan para clasificar la corrosión (11) es el medio fisicoquímico en el que ocurre el ataque. Si el ataque se produce por especies fundidas se denomina "corrosión por sales fundidas a alta temperatura"; y si se produce por los gases presentes en el ambiente de trabajo se conoce como "corrosión por gases calientes". La corrosión por especies fundidas es el mecanismo dominante de degradación cuando el combustible utilizado es de los denominados "sucios", como el gas-oil. Los contaminantes adicionales, como el sodio, pueden dar lugar a la formación de sales de sulfato sódico $\left(\mathrm{Na}_{2} \mathrm{SO}_{4}\right)$, con un punto de fusión inferior a la temperatura de trabajo del material cerámico, pudiendo producir modificaciones estructurales y de fases al penetrar por los poros del material hacia las zonas internas del mismo. Este es el caso de la degradación que sufren los TBCs fabricados con $\mathrm{ZrO}_{2}$ estabilizado con $7 \%$ en peso de $\mathrm{Y}_{2} \mathrm{O}_{3^{\prime}}$ que se utilizan en las turbinas operadas con gas-oil (12-14) en las que, entre otras, se produce la siguiente reacción:

$$
\begin{aligned}
& \mathrm{ZrO}_{2}(\text { tetragonal })\left(\mathrm{Y}_{2} \mathrm{O}_{3}\right)+\mathrm{Na}_{2} \mathrm{SO}_{4} \rightarrow \\
& \mathrm{ZrO}_{2}(\text { monoclinica })+2 \mathrm{NaYO}_{2}+\mathrm{SO}_{3}
\end{aligned}
$$

Esta reacción da lugar a la transformación de $\mathrm{ZrO}_{2}$ tetragonal a monoclínica con el consiguiente aumento de volumen.

Por otro lado, los combustibles gaseosos tienen un menor contenido en impurezas que la gasolina o el gas-oil y, por tanto, las especies que pueden producir la corrosión del material son distintas y los mecanismos de degradación que se producen también. En este caso, la atmósfera de combustión, además de caracterizarse por la presencia de $\mathrm{CO}, \mathrm{CO}_{2^{\prime}} \mathrm{NO}$ y $\mathrm{NO}_{2}$ perjudiciales para el ser humano, también contiene $\mathrm{H}_{2}, \mathrm{H}_{2} \mathrm{O}$, $\mathrm{O}_{2}$, y especies transitorias como el $\mathrm{OH}$, y $\mathrm{H}$, que podrían afectar al material que soporta la combustión. Dependiendo de la naturaleza de éste, unas especies serán más agresivas que otras y los mecanismos que gobiernan la degradación en cada caso serán distintos. En el caso de los materiales cerámicos oxídicos, los mecanismos estarán relacionados con la reducción del material, mientras que en el caso de los materiales no oxídicos el mecanismo principal será la oxidación. La concentración de las especies agresivas es un parámetro fundamental en la cinética de reacción.

Un mecanismo de degradación común a materiales oxídicos y no oxídicos con base silicio es la volatilización de la sílice, que se encuentra dentro de la estructura de los primeros o formando una capa superficial, producto de la oxidación, en los estos últimos (15). Se sabe que la interacción del $\mathrm{SiO}_{2}$ con la atmósfera de combustión está directamente relacionada con la degradación de estos materiales (16). $\mathrm{El} \mathrm{SiO}_{2}$ puede reaccionar con el hidrógeno y el CO presentes en la atmósfera de combustión, formando $\mathrm{SiO}(\mathrm{g})$ que se volatiliza.

$\mathrm{SiO}_{2}+\mathrm{H}_{2}(\mathrm{~g}) \rightarrow \mathrm{SiO}(\mathrm{g})+\mathrm{H}_{2} \mathrm{O}(\mathrm{g})$

$\mathrm{SiO}_{2}+\mathrm{CO}(\mathrm{g}) \rightarrow \mathrm{SiO}(\mathrm{g})+\mathrm{CO}_{2}(\mathrm{~g})$

Si la atmósfera contiene vapor de agua, el $\mathrm{SiO}_{2}$ puede además reaccionar con $\mathrm{el} \mathrm{H}_{2} \mathrm{O}$ para formar hidróxidos volátiles (17):

$\mathrm{SiO}_{2}+2 \mathrm{H}_{2} \mathrm{O}(\mathrm{g}) \rightarrow \mathrm{Si}(\mathrm{OH})_{4}(\mathrm{~g})$

Las altas presiones (6-10 atm) y velocidades de los gases $(18-24 \mathrm{~m} / \mathrm{s})$ en los motores de turbina favorecen este mecanismo de degradación; sin embargo, las condiciones de trabajo de las calderas de combustión domésticas están muy alejadas de dichas condiciones.

Finalmente, cuando los componentes cerámicos trabajan en condiciones de ciclado térmico aparecen tensiones residuales asociadas a las diferencias en los coeficientes de expansión térmica entre los distintos componentes, que contribuyen a la degradación del material. En el caso de los quemadores cerámicos su estructura macroporosa atenúa este efecto. Sin embargo, estas diferencias de expansión térmica con la temperatura pueden ser importantes en el caso de las barreras térmicas, especialmente para las llamadas TBCs gruesas con capas cerámicas que superan $1 \mathrm{~mm}$. Diferentes estudios de laboratorio han demostrado la aparición de grietas, tanto perpendiculares como paralelas al substrato, durante el ciclado, que pueden llegar a producir el fallo del TBC por delaminación $(8,10,18)$. Por otro lado, la sinterización parcial de la capa exterior del recubrimiento, que está sometida a más elevada temperatura durante el servicio, contribuye al aumento de las tensiones térmicas y acentúa la degradación en este tipo de sistemas (19).

En este trabajo se presentan los resultados del estudio de degradación de dos materiales cerámicos en dos aplicaciones distintas. Uno de ellos es un material de 
cordierita $\left(2 \mathrm{MgO} \cdot 2 \mathrm{Al}_{2} \mathrm{O}_{3} \cdot 5 \mathrm{SiO}_{2}\right)$ empleado como quemador de gas radiante en una cámara de combustión, que modeliza de forma acelerada el comportamiento en calderas de combustión domesticas operadas con gas natural. El otro es un recubrimiento grueso de $1,5 \mathrm{~mm}$ de espesor de $\mathrm{ZrO}_{2}$ estabilizado con $7 \%$ en peso de $\mathrm{Y}_{2} \mathrm{O}_{3}$. Este recubrimiento funciona como capa superior de un sistema TBC en una turbina de una central térmica de ciclo combinado, que utiliza como combustible gas-oil. En los siguientes apartados se describirán los efectos que la atmósfera de combustión produce sobre la microestructura, propiedades y composición de fases cristalinas de ambos materiales, dando una explicación razonada de los tipos de degradación que se producen.

\section{ESTUDIO DE LA DEGRADACIÓN EN ATMÓSFERAS DE COMBUSTIÓN}

\subsection{Quemadores de gas}

Los quemadores de gas utilizados en este estudio están comercializados por Morgan Matroc (ahora Rauschert España S.L.) y son del tipo "placa perforada", de forma rectangular $\left(13 \times 16 \times 1,5 \mathrm{~cm}^{3}\right)$, con miles de canales de $\sim 1 \mathrm{~mm}$ de diámetro distribuidos ordenadamente por la superficie (Fig. 1). La porosidad de la placa es $70 \%$ (vol.) y su composición corresponde a la cordierita, con un contenido de impurezas de óxidos de hierro y titanio menor del $1 \%$ en peso.

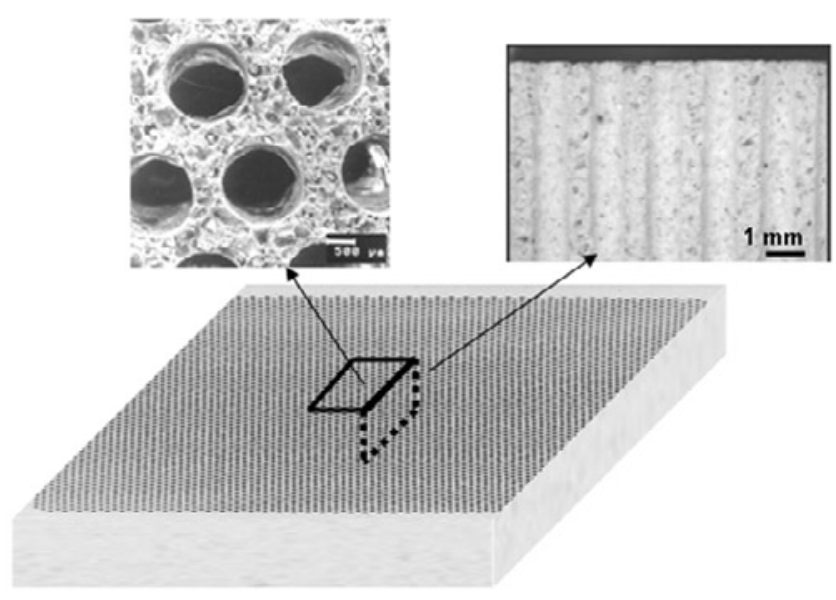

Fig. 1- Detalle de quemador cerámico tipo "placa perforada" producido por Morgan Matroc S.A. (ahora Rauschert España).

Este tipo de quemadores se envejecieron en Cámaras de Combustión Estandarizadas desarrolladas por Ikerlan S. A. (Vitoria, España), usando gas natural como combustible. Las cámaras consistían básicamente en un combustor y un sistema de control que permitía el control automático de los parámetros de combustión. Estas cámaras pueden reproducir las condiciones de trabajo que se dan en calderas utilizadas en aplicaciones domésticas. El gas y el aire se mezclaban en una precámara antes de pasar a través de los canales de la placa cerámica y alcanzar la zona superior de la misma donde la mezcla se inflamaba. Dos de las paredes de la cámara de combustión tenían ventanas desde donde se podía controlar visualmente la combustión (Fig. 2). Además, la temperatura y otros parámetros de la combustión (concentración de $\mathrm{O}_{2^{\prime}}$ $\mathrm{CO}_{x}$ y $\mathrm{NO}_{x}$ ) se medían mediante distintos sensores y eran registrados por el sistema de control.

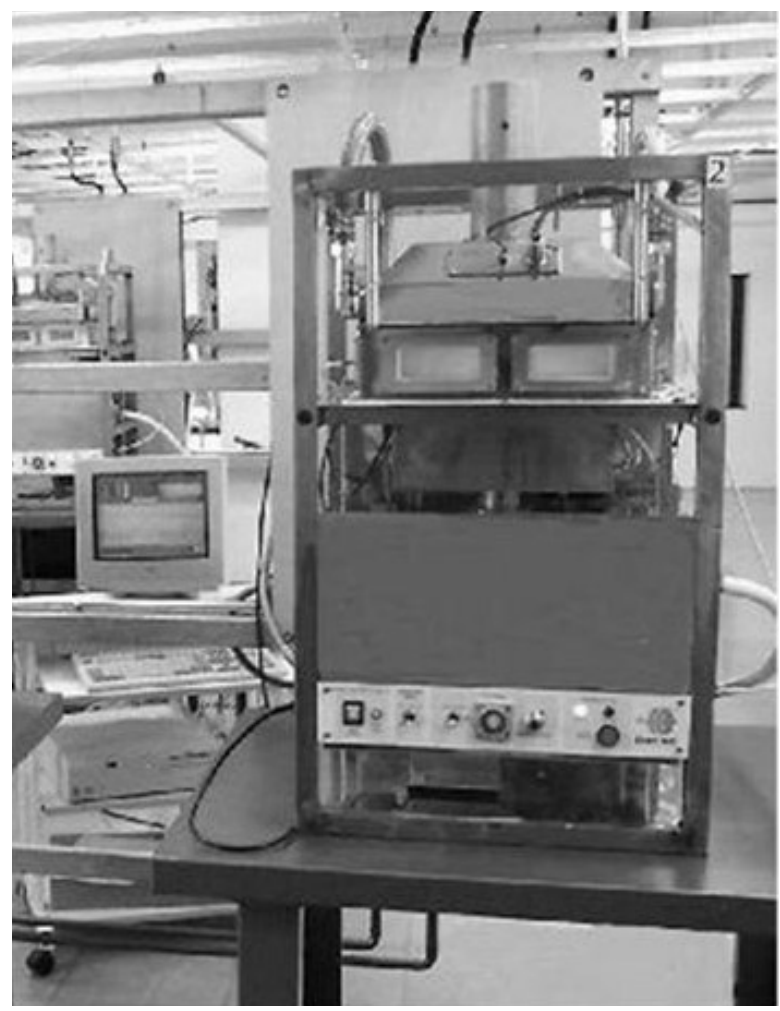

Fig. 2- Cámara de combustión estandarizada desarrollada por Ikerlan S.A.

Los ensayos de envejecimiento consistieron en ciclos de encendido/apagado cuya duración variaba entre 75 y $3600 \mathrm{~s}$ (con un tiempo fijo de apagado de $45 \mathrm{~s}$ ). El tiempo máximo que una placa fue sometida a estos ciclos de degradación fue de $3500 \mathrm{~h}$ trabajando en modo radiante. Durante los ensayos, la temperatura de la zona superior de la placa, donde se producía la combustión, era del orden de $900^{\circ} \mathrm{C}$, mientras que las temperaturas de la zona inferior no sobrepasaban $\operatorname{los} 100^{\circ} \mathrm{C}$. Hay que destacar que durante ningún ensayo se sobrepasaron los límites de emisión de contaminantes que pudieran indicar que se estuviera produciendo una mala combustión. Es decir, en este aspecto no había señales que indicaran degradación en las placas.

Los cambios en la composición de las fases de las placas envejecidas se analizaron, usando difracción de rayos $\mathrm{X}$ (DRX) con un ángulo de incidencia rasante, en capas cada vez más profundas mediante el desbastado secuencial de la placa. En la Fig. 3 está representada una selección de los difractogramas obtenidos. La espinela de $\mathrm{Al}_{2} \mathrm{O}_{3}-\mathrm{MgO}\left(2 \mathrm{Al}_{2} \mathrm{O}_{3} \cdot \mathrm{MgO}\right)$ es la fase principal encontrada en la superficie de combustión, aunque en esta capa también se detectan picos asociados a la cordierita $\left(2 \mathrm{MgO} .2 \mathrm{Al}_{2} \mathrm{O}_{3} .5 \mathrm{SiO}_{2}\right)$ y la forsterita $\left(2 \mathrm{MgO} \mathrm{SiO}_{2}\right)$. En las capas interiores de la muestra se produce un decrecimiento progresivo de la intensidad de los picos asociados a la espinela y la forsterita, mientras que la intensidad de los picos asociados a la cordierita crece. A una profundidad de $\approx 160 \mu \mathrm{m}$ el espectro obtenido coincide con el de la placa sin degradar. 


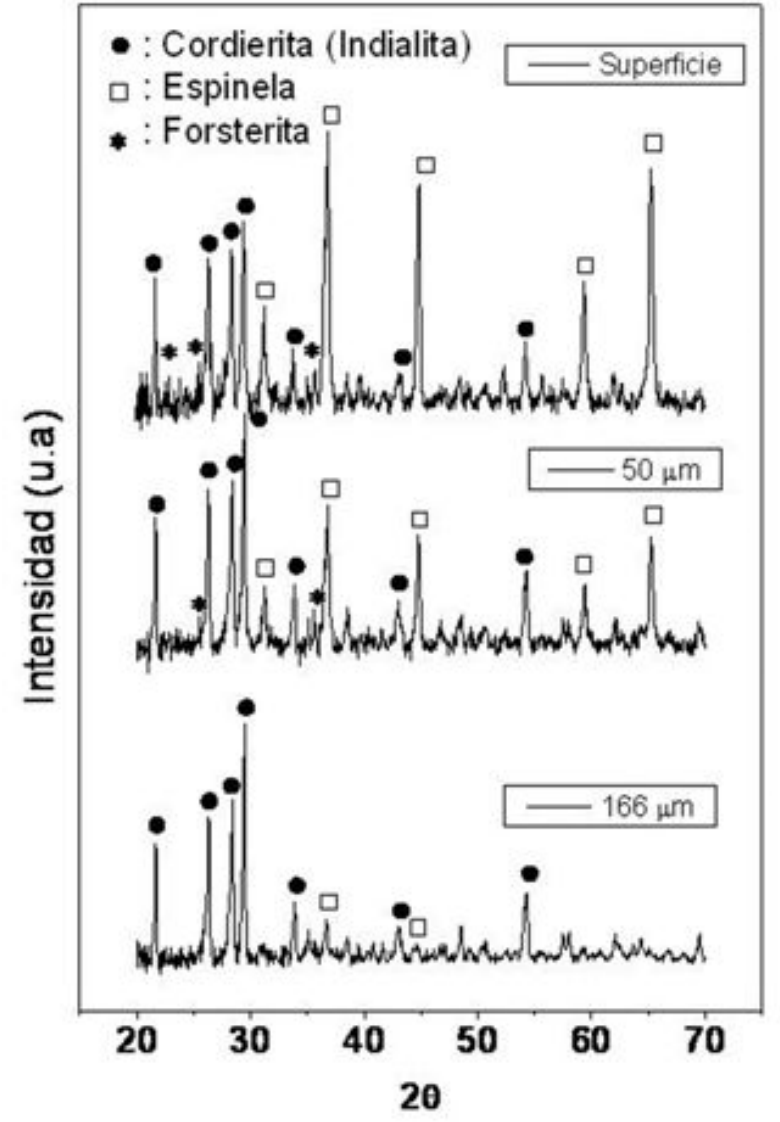

Fig. 3- Difractogramas DRX, obtenidos en condiciones de ángulo de incidencia rasante, para una placa envejecida a diferentes profundidades (mostradas en la figura) medidas desde la superficie de combustión.

El análisis microestructural de las placas realizado por microscopía electrónica de barrido (MEB) reveló que existía una perdida de material en las zonas próximas a la superficie de combustión, mientras que en las zonas más internas la microestructura estaba recubierta por fase vítrea (Fig. 4). Mediante análisis por energía dispersada de rayos X (EDX) en las distintas zonas, se pudo observar que la composición $\mathrm{MgO}-\mathrm{Al}_{2} \mathrm{O}_{3}-\mathrm{SiO}_{2}$ en la superficie de la placa envejecida se desplazaba desde la inicial (composición promedio $51 \% \mathrm{SiO}_{2^{\prime}}$ $35 \% \mathrm{Al}_{2} \mathrm{O}_{3}$ y $14 \% \mathrm{MgO}$ ) hacia el campo de cristalización de la cordierita, la espinela y la forsterita (composición promedio $27 \% \mathrm{SiO}_{2}, 52 \% \mathrm{Al}_{2} \mathrm{O}_{3}$ y $21 \% \mathrm{MgO}$ ), del sistema $\mathrm{MgO}-\mathrm{Al}_{2} \mathrm{O}_{3}-$ $\mathrm{SiO}_{2}$ (Fig. 5). Estas tres fases son las mismas que se detectaban mediante DRX en la capa superior de las placas envejecidas (20). Es interesante resaltar que el punto invariante para la composición de la capa exterior es $1370^{\circ} \mathrm{C}$, frente a $1460^{\circ} \mathrm{C}$ de la composición inicial, lo que indica una disminución de la refractariedad de la superficie de la placa. Esta degradación superficial se ha comprobado que aumenta con el tiempo de total de ensayo y afecta a las propiedades mecánicas de la placa, en concreto al modulo de elasticidad y a la resistencia mecánica (20).

La modificación de la microestructura y propiedades del material que forma los quemadores debe estar causada por las altas temperaturas y la presencia de especies químicas transitorias características de la combustión del metano $(\mathrm{CO}$,

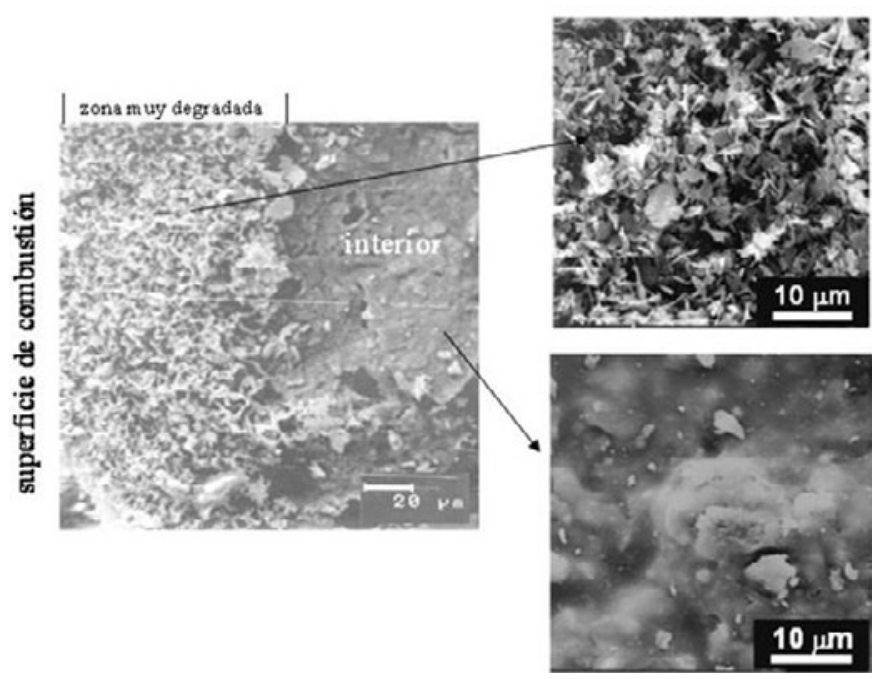

Fig. 4- Micrografía MEB de una zona próxima a la superficie de combustión con detalles de la zonas más degradadas y de la zona interior sin degradar.

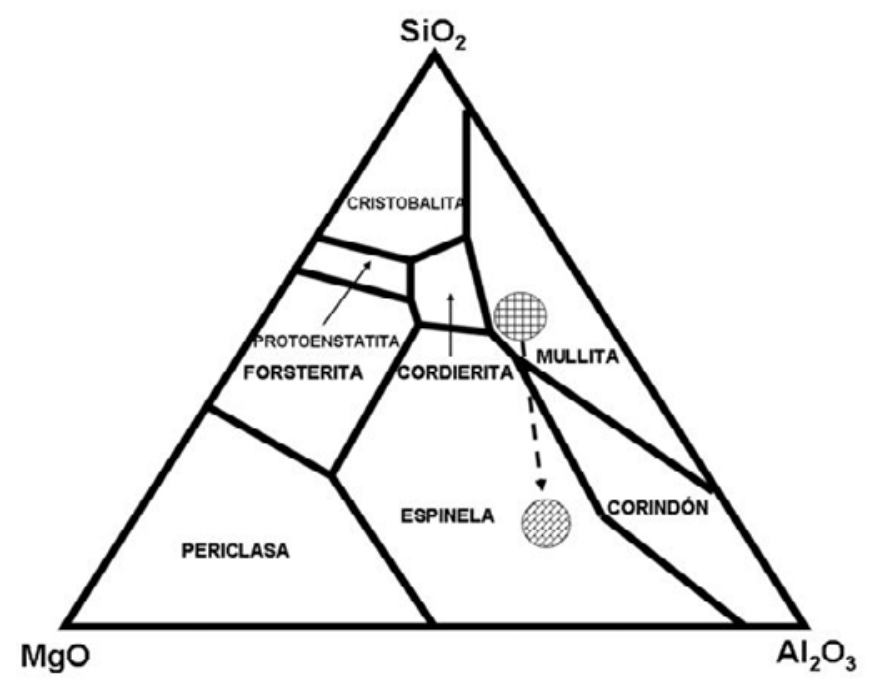

Fig. 5- Diagrama de equilibrio de fases (21) donde se muestra el cambio de composición que se produce en la superficie de combustión.

$\mathrm{OH}, \mathrm{H})$ (22). Entre los materiales cerámicos oxídicos, la sílice y los materiales que contienen $\mathrm{SiO}_{2}$ en su composición, como es el caso de la cordierita, exhiben una pobre estabilidad en atmósferas reductoras (23). De forma particular, la sílice puede reducirse por $\mathrm{CO}, \mathrm{H}_{2} \mathrm{o} \mathrm{H}$, como se indicó en la introducción de este trabajo, siendo esta última especie la que posee un mayor poder reductor $(23,24)$.

$$
\frac{1}{2} \mathrm{SiO}_{2}+\mathrm{H}(\mathrm{g}) \rightarrow \frac{1}{2} \mathrm{SiO}(\mathrm{g})+\frac{1}{2} \mathrm{H}_{2} \mathrm{O}(\mathrm{g})
$$

De igual forma, podemos formular reacciones de descomposición de corderita en hidrógeno monoatómico, 
asumiendo la formación de espinela, tal y como se ha observado por DRX:

$$
\begin{aligned}
& \frac{1}{10}\left(2 \mathrm{MgO} \cdot \underset{\text { cordierita }}{\left.2 \mathrm{Al}_{2} \mathrm{O}_{3} \cdot 5 \mathrm{SiO}_{2}\right)+\mathrm{H}(\mathrm{g}) \rightarrow}\right. \\
& \frac{1}{5} \underset{\text { espinela }}{\left(\mathrm{MgO} \cdot \mathrm{Al}_{2} \mathrm{O}_{3}\right)}+\frac{1}{2} \mathrm{SiO}(\mathrm{g})+\frac{1}{2} \mathrm{H}_{2} \mathrm{O}(\mathrm{g})
\end{aligned}
$$

Mediante cálculos termodinámicos realizados con el programa Outokumpu HSC Chemistry (Versión 5.1) se verificó que esta reacción es termodinámicamente favorable para temperaturas de $900^{\circ} \mathrm{C}$ o mayores, que coincide con las que se alcanzan en la superficie del quemador cuando trabaja en modo radiante (20). La extensión de esta zona de corrosión está limitada a las proximidades de las superficie del quemador $(\sim 150 \mu \mathrm{m})$ por la rápida caída de temperatura que se produce hacia el interior del quemador, y porque la concentración de radicales libres es sólo apreciable en zona cercana a la superficie de combustión (22).

A la vista de estos resultados, se puede deducir que la degradación de los quemadores cerámicos de cordierita que operan con gas natural en modo radiante es del tipo "corrosión por gases calientes", y afecta a un pequeño volumen de material próximo a la superficie de combustión.

2.2 Degradación de barreras térmicas en motores de turbina

En este apartado se presentan los resultados del estudio realizado sobre una barrera térmica degradada en servicio en la cámara de combustión de la turbina de gas de una central térmica. Esta turbina utiliza gas-oil como combustible y no funciona en continuo, sino que lo hace en ciclos de encendido y apagado según las exigencias de potencia. Las temperaturas de los gases en la zona donde se encuentra la placa analizada pueden alcanzar los $1400^{\circ} \mathrm{C}$.

El análisis de microscopía óptica de luz reflejada (MOLR) realizado en las zonas degradadas de la placa reveló la presencia de grietas paralelas a la superficie del substrato (Fig. 6). Las grietas se observan tanto dentro de la capa de anclaje (CA) como en el interior del recubrimiento cerámico. En algunas zonas estas grietas se propagaron hasta producir la delaminación parcial del recubrimiento.

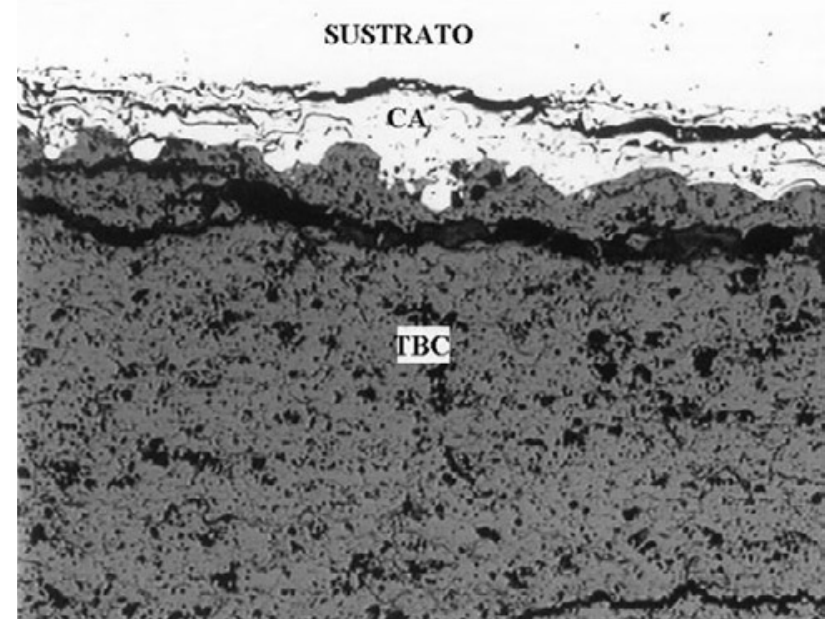

Fig. 6- Detalle obtenidos mediante MOLR de una zona degradada.
El sistema estudiado es el típico utilizado en estas aplicaciones (18), y estaba compuesto por un substrato metálico de base $\mathrm{Ni}$, una capa de anclaje de composición $\mathrm{MCrYAl}(\mathrm{M}=\mathrm{Ni}$ o NiCo) y, finalmente, un recubrimiento cerámico de $\mathrm{ZrO}_{2}$ estabilizado con $\mathrm{Y}_{2} \mathrm{O}_{3}$ (Fig. 7a). Como se puede observar en las micrografias obtenidas por MEB de la Figura 7, la porosidad de la capa aumenta hacia el interior, lo que indica que se ha producido cierta densificación durante el uso. Tanto en la intercara sustrato/CA (Fig. 7b) como en la intercara CA/TBC (Fig. 7c) se detecta la presencia de fases con distinta coloración. El recubrimiento cerámico propiamente dicho, mostrado en la Fig. $7 d$, presenta la microstructura típica de los recubrimientos fabricados mediante proyección por plasma, en la que se puede observar porosidad esférica y también alargada, así como líneas más curvas que indican la separación entre los "splats" (gotas fundidas y aplastadas contra el sustratos), y también otros defectos de gran tamaño. Esta microestructura favorece la disminución de la conductividad térmica y de la rigidez del recubrimiento.
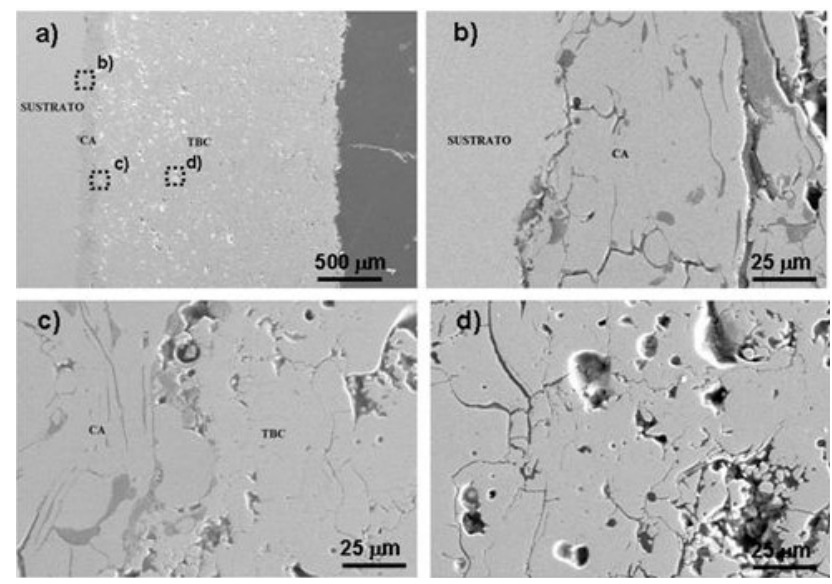

Fig. 7- Micrografía de MEB de una sección transversal de la placa degradada con distintos detalles a mayores aumentos de las intercaras sustrato/CA (b) y CA/TBC (c) y de la región de la TBC (d).

También, para determinar los cambios de composición, se realizaron análisis por energía dispersada de rayos X (EDX) en las zonas más degradadas, en las que se había producido un desprendimiento parcial del recubrimiento cerámico, (Fig. 6). Así, en la intercara substrato metálico/capa de anclaje, generalmente se detectaban granos prismáticos de color gris oscuro (Fig. 8), con un tamaño aproximado de $10 \mu \mathrm{m}$, cuyo espectro EDX permite identificarlos como $\mathrm{Al}_{2} \mathrm{O}_{3}$. Dichos granos suelen estar asociados con zonas alteradas en el interior de la CA cerca de la TBC, como las señaladas con una $\mathrm{R}$ en la Fig 8. Los espectros EDX de estas zonas muestran la presencia mayoritaria de $\mathrm{Cr}$, Al y Si y, por lo tanto, corresponderían con óxidos mixtos de $\mathrm{Cr}, \mathrm{Al}$, Si o bien composiciones tipo espinela. En esta zona además se detecta un porcentaje significativo de azufre $(\sim 13 \%$ peso $)$ y también de $\mathrm{Na}(\sim 3 \%$ peso), que deben provenir del combustible. Además, también se detectan otras fases con composiciones binarias de Al-Y y de S-Cr, Xl Lo que puede indicar la presencia de aluminatos de itrio y sulfatos de cromo, que se producirían en lugar de la capa continua protectora de $\mathrm{Al}_{2} \mathrm{O}_{3}$ crecida térmicamente (8). Por otro lado, los análisis MEB-EDX realizados sobre la superficie 
de fractura de los trozos desprendidos muestran la formación de cristalizaciones en forma de pequeñas agujas (Fig 9) que contienen $\mathrm{Cr}$, $\mathrm{Al}$ y una pequeña fracción de $\mathrm{S}$. La formación de estas agujas indicaría la existencia de reacciones en fase sólido-vapor.

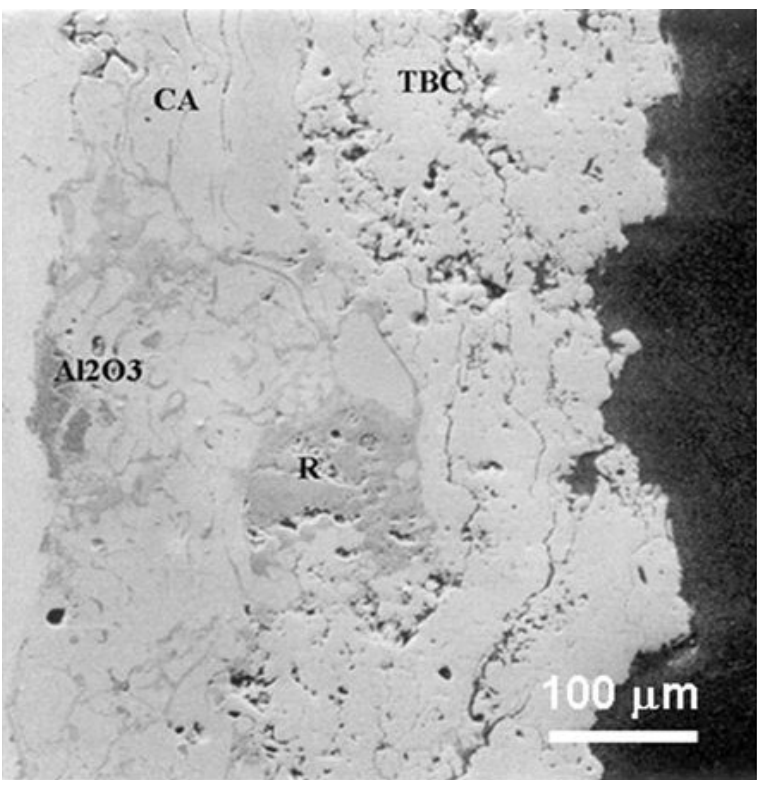

Fig. 8- Microestructura de la zona más degradada de la placa donde se observa la delaminación parcial del recubrimiento a una distancia de $\sim 100 \mu \mathrm{m}$ de la intercara CA/recubrimiento cerámico. $\mathrm{R} \equiv$ zona de reacción.

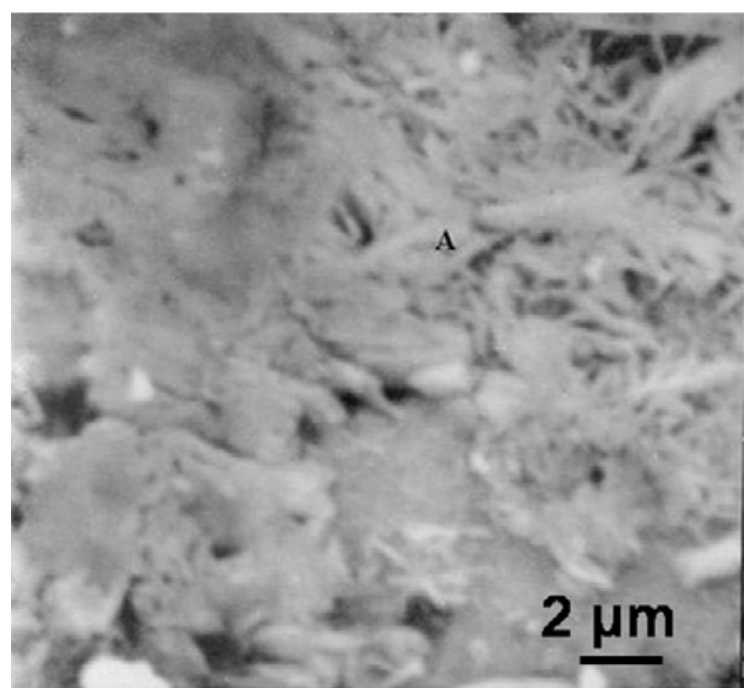

Fig. 9- Detalle de la superficie de fractura de una muestra delaminada, donde se ven cristalizaciones (A) con forma de aguja que presentan contenidos significativos en $\mathrm{Cr}, \mathrm{Al}$, y $\mathrm{S}$.

Por último, la presencia de contaminantes del combustible se confirmó mediante análisis de fluorescencia de rayos $X$ (FRX), realizados en las dos caras de una muestra de TBC desprendida durante el mecanizado; i.e., en la superficie interna en contacto con la CA y en la superficie externa en contacto con los gases calientes. En la Fig. 10 se puede observar como existe una importante impurificación por sodio y azufre en la cara interna, comparado con la superficie externa, lo que demuestra que estos contaminantes han penetrado a través de la capa cerámica.
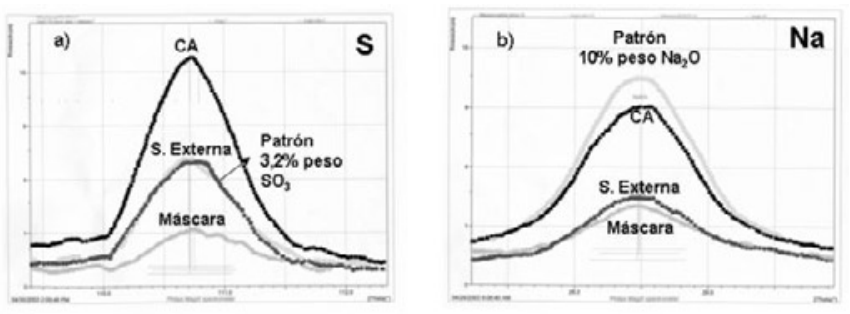

Fig. 10- Análisis FRX de contenido en Azufre (a) y Sodio (b) realizados en muestras tomadas de las zonas en contacto con la capa de anclaje (CA) y de la superficie externa (S. Externa) de fragmentos de recubrimiento desprendido durante el mecanizado. También se muestran los resultados obtenidos para la máscara y patrones de contenido de azufre y sodio conocido.

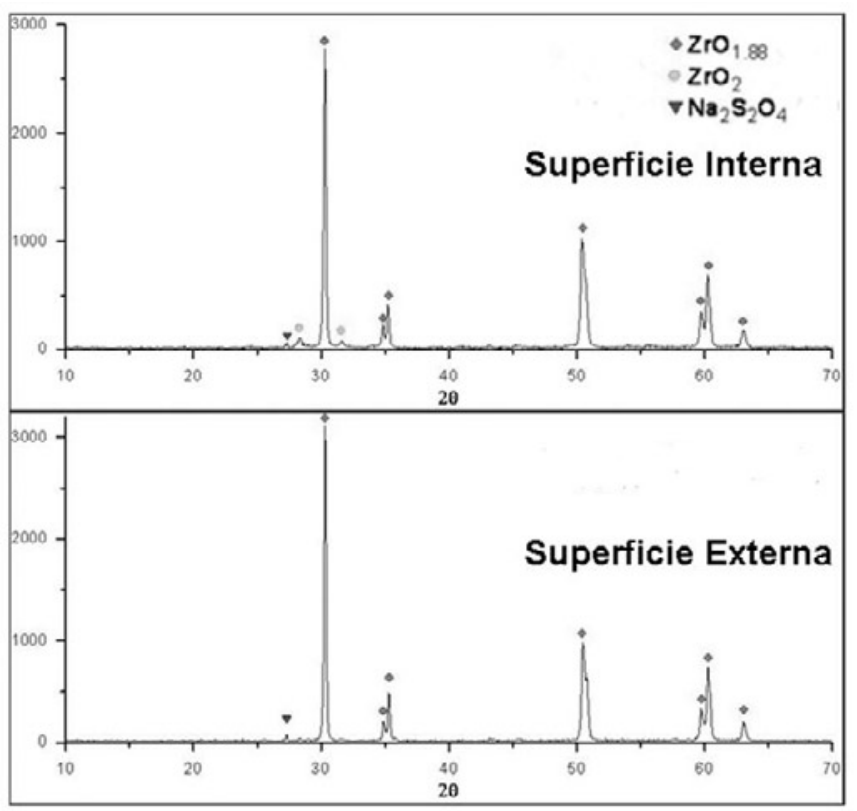

Fig. 11- Difractogramas de rayos $\mathrm{X}$ obtenidos para las dos superficies la TBC desprendida de la placa y analizadas por DRX.

En la Fig. 11 se representan los espectros de DRX correspondientes a las mismas superficies analizadas por FRX. La fase mayoritaria en ambas es $\mathrm{ZrO}_{188}$ tetragonal, no estequiométrica. En la superficie interna se aprecia también la presencia de una pequeña cantidad de $\mathrm{ZrO}_{2}$ monoclínica ( $10 \%$ en peso), que no se detectó en la superficie exterior, lo que indica una desestabilización de la fase de $\mathrm{ZrO}_{2}$-tetragonal. Además, en ambos difractogramas se detecta un pequeño pico que se podría atribuir al $\mathrm{Na}_{2} \mathrm{~S}_{2} \mathrm{O}_{4}$. No se han podido detectar otros productos de corrosión debido probablemente a que se encuentran en un porcentaje menor al límite de detección de la técnica $(\approx 5 \%$ en peso).

La degradación parece que se produce por un mecanismo de "corrosión por sales fundidas". En la atmósfera de trabajo se han formado sales de $\mathrm{Na}$ y $\mathrm{S}$ de muy bajo punto de fusión, que han penetrado hacia el interior por las grietas y los poros, y han producido la degradación de la capa de anclaje. Como consecuencia, la capa continua de $\mathrm{Al}_{2} \mathrm{O}_{3}$ no se desarrolla y se forman óxidos de cromo, óxidos mixtos tipo 
espinela, aluminatos de itrio, sulfatos de cromo y sulfatos de sodio. Los resultados obtenidos difieren del comportamiento esperado durante la puesta en servicio de este tipo de recubrimientos, en que se genera una capa fina y continua de $\mathrm{Al}_{2} \mathrm{O}_{3}$ entre la $\mathrm{CA}$ y la TBC, que debiera proteger el sustrato frente a la oxidación. Esta capa puede comprometer el comportamiento mecánico de las barreras térmicas debido al desarrollo de tensiones térmicas residuales, especialmente cuando su espesor sobrepasa un cierto límite $(\approx 10 \mu \mathrm{m})$ (8). Existen algunos trabajos en la literatura que refieren la formación de otros óxidos y espinelas de Ni o Co $(25,26)$, cuando no existe suficiente $\mathrm{Al}$ disponible en la CA para

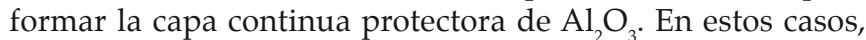
el itrio de la capa de anclaje, capta el azufre corrosivo presente en las atmósferas de combustión (27), reacciona con el aluminio y, por lo tanto, deja de ser efectivo como barrera antioxidante. Por otro lado, el sulfato sódico fundido ácido puede reaccionar con el itrio que estabiliza el $\mathrm{ZrO}_{2}$, lo que explicaría la desestabilización de la fase $\mathrm{ZrO}_{2}$-t y la presencia de $\mathrm{ZrO} 2-\mathrm{m}$ detectada por DRX. La transformación $\mathrm{ZrO}_{2}-\mathrm{t}$ $\rightarrow \mathrm{ZrO}_{2}-\mathrm{m}$ lleva asociada un aumento de volumen del $4 \%$, lo que da lugar a un incremento de las tensiones en la zona y contribuye a la formación de grietas y a la delaminación del TBC. Finalmente, el aumento de densidad observado en la capa más externa del TBC producido durante el servicio también puede contribuir a un aumento de las tensiones residuales de origen térmico durante el ciclado. Esto explicaría el hecho observado de que la delaminación se produzca en el interior del recubrimiento cerámico, a una cierta distancia de la intercara con la CA.

\section{CONCLUSIONES}

Se han analizado los mecanismos de degradación de materiales cerámicos empleados en dos aplicaciones tecnológicas de alta temperatura. En el caso de materiales cerámicos empleados como quemadores radiantes, se ha demostrado que la degradación se produce por corrosión a alta temperatura en atmósfera parcialmente reductora mientras que en el caso de los recubrimientos cerámicos, empleados como barreras térmicas de turbinas de gas, es el de corrosión por sales fundidas. En el primer caso, la degradación del material es debida a la reducción preferente del $\mathrm{SiO}_{2}$ en el compuesto por las especies intermedias que se producen en las reacciones de combustión y está localizada en un pequeño volumen cercano a la superficie de combustión. En caso de la TBC, la degradación se debe a la presencia de Na y S en la atmósfera de combustión, ya que forman sales de bajo punto de fusión que penetran a través de bordes de grano, poros y grietas hacia las zonas internas del sistema alcanzando la capa metálica de anclaje.

\section{AGRADECIMIENTOS}

Los autores agradecen la financiación al Ministerio de Educación y Ciencia (Proyecto MEC-MAT2006-07118). Eugenio García agradece la financiación de este estudio al programa I3P-PC2005L.

\section{REFERENCIAS}

1. I. M. Lachman, R. N. McNally, Monolithic honeycomb supports for catalysis, Chem. Eng. Progress, 81 29-31 (1985).

2. R. Heck, R. Ferrauto, Atomotive catalysts, Automotive Engineering, 93-96, (1996).

3. M. Arai, K. Amagai, T. Mogi, Catalytic combustion of pre-vaporized liquid fuel, J. Energy Resources Tech., 123, 44-49 (2001).

4. J. K. Kilham and E. P. Laningan, A study of the mechanism of radiant burners Gas Council Research Communication GC167, I. G. E. Journal 700713 (1970).

5. L. Ticó, Infrared - clean and efficient heat at the speed of light, Global Ceramic Review 4/98, 12 (1998/99).

6. E. García, P. Miranzo, M. I. Osendi, Quemadores de gas cerámicos, Bol. Soc. Esp. Ceram. V. 42, 5, 277-282 (2003).

7. D.R. Clarke, C.G. Levi, Materials Design for the Next Generation Thermal Barrier Coatings, Annu. Rev. Mater. Res., 33, 383-417 (2003).

8. N.P Padture, M. Gell and E.H. Jordan, Thermal barrier coatings for gasturbine engine applications, Science, 296, 280-284 (2002)

9. D.W. Parker, Thermal barrier coatings for gas turbines, automotive engines and diesel engines, Materials \& Design, 13, 6, 345-351 (1992).

10. A. G. Evans, D. R. Mumm, J. W. Hutchinson, G. H. Meier, and F. S. Petit, Mechanisms controlling the durability of thermal barrier coatings, Prog. Mater. Sci., 46, 505-553 (2001).

11. K. G. Nickel, Corrosion of non-oxide ceramics, Ceram. Inter., 23, 127-133, (1995).

12. S.Y. Park, J.H. Kim, M.C. Kim, H.S. Song, C.G. Park, Microscopic observation of degradation behaviour in yttria and ceria stabilized zirconia thermal barrier coatings under hot corrosion, Surf. Coat. Technol. 190, 357365 (2005).

13. C. Batista, A. Portinha, R.M. Riberiro, V, Teixeira, C.R. Oliveira, Evaluation of laser-glazed plasma-sprayed thermal barrier coatings under high temperature exposure to molten salts, Surf. Coat. Technol. 200, 6783-6791 (2006).

14. A. Afrasiabi, M. Saremi, A. Kobayashi, A comparative study on hot corrosion resistance of three types of thermal barrier coatings: YSZ, $\mathrm{YSZ}+\mathrm{Al}_{2} \mathrm{O}_{3}$ and $\mathrm{YSZ} / \mathrm{Al}_{2} \mathrm{O}_{3}$, Mater. Sci. Eng. A, 478, 264-269 (2008).

15. N. J. Shaw y col. Materials for engine applications above $3000^{\circ} \mathrm{F}$-an overview, NASA Rept. No. TM-100169, NASA Lewis Research Center, Cleveland, OH, (1987).

16. N. S. Jacobson, Corrosion of silicon-based ceramics in combustion environments, J. Am. Ceram. Soc., 76, 1, 3-28 (1993).

17. E. J. Opila, J. L. Smialek, R. C. Robinson, D. S. Fox y N. S. Jacobson, $\mathrm{SiC}$ recession caused by $\mathrm{SiO}_{2}$ volatility under combustion conditions: II, thermodynamics and gaseous-diffusion model, J. Am. Ceram. Soc., 82, 7, 1826-34 (1999).

18. D. Schwingel, R. Taylor, T. Haubold, J. Wigren, C. Gualco, Mechanical and thermophysical properties of thick PYSZ thermal barrier coatings: correlation with microstructure and spraying parameters, Surf. Coat. Technol. 108-109, 99-106 (1998).

19. B.D. Choules, K. Kokini, T. A. Taylor, Thermal fracture of thermal barrier coatings in a high heat flux environment, Sur. Coat. Technol. 106, 23-29 (1998).

20. E. García, P. Miranzo y M. I. Osendi, Alterations in cordierite based burners subjected to radiant mode ageing conditions, J. Euro. Ceram. Soc., 23, 30473053 (2003).

21. W. Schreyer, J. F. Schairer, “Composition and structural states of anhydrous $\mathrm{Mg}$-cordierites: a re-investigation of the system $\mathrm{MgO}-\mathrm{Al}_{2} \mathrm{O}_{3}-\mathrm{SiO}_{2}$, J. Petrol., 2, 324-406 (1961).

22. P. H. Bouma, Methane-air Combustion on Ceramic Foam Surface Burners, Memoria de Tesis Doctoral. Eindhoven University of Technology, (1997).

23. J. L.Lannutti, R. A. Rapp, Degradation of radiant ceramics structures in combustion atmospheres, GRI-reports 92/0408, 1992 and 93/0435 (1994).

24. G. Wang, J. L. Lannutti, Surface cavity microenvironments in a porous ceramic radiant burner, Combust. Sci. And Tech., 95, 277-311 (1994)

25. D.R. Mumm, A.G. Evans, On the role of imperfections in the failure of a thermal barrier coating made by electron beam deposition, Acta Mater. 48, 8, 1815-1827 (2000).

26. E.Y Lee, R.R. Biederman, R.D. Sisson Jr., Diffusional interactions and reactions between a partially stabilized zirconia thermal barrier coating and the NiCrAlY bond coat, Mater. Sci. Eng. A. 121, 467 (1989)

27. J.L. Smialek, D.T. Jayne, J.C. Schaeffer,W.H. Murphy, Effects of hydrogen annealing, sulfur segregation and diffusion on the cyclic oxidation resistance of superalloys: a review, Thin Solid Films, 253, 1-2, 285-292 (1994).

Recibido: $\quad 16.04 .08$

Aceptado: 15.07 .08 